\title{
On A New Class of Numbers
}

\author{
${ }^{1}$ P. Rajkhowa, ${ }^{2}$ Ananta Kumar Bora \\ Department of Mathematics Gauhati University Guwahati-781014,India
}

\begin{abstract}
The present paper studies a new class of numbers. Results obtained in this paper are a table, recurrence relations, generating functions and Summation formulas for these new class of numbers . Many results reduce to their corresponding results for the Catalan numbers .
\end{abstract}

(1.1) Consider the quadratic equation

\section{Definition:}

\section{$\mathrm{x}^{2}=2 \mathrm{x}+1$}

Its roots are

$\alpha=1+\sqrt{2}$

$\beta=1-\sqrt{2}$

$\alpha+\beta=2$

$\alpha \beta=-1$

Let $\mathrm{V}_{\mathrm{n}}=\alpha^{\mathrm{n}}+\beta^{\mathrm{n}}$ for $\mathrm{n} \geq 0$, then

$\mathrm{V}_{0}=2, \mathrm{~V}_{1}=2, \mathrm{~V}_{2}=6, \mathrm{~V}_{3}=14, \mathrm{~V}_{4}=34, \mathrm{~V}_{5}=82, \mathrm{~V}_{6}=198, \mathrm{~V}_{7}=478$,

$\mathrm{V}_{8}=1154$ etc.

These numbers satisfies the following recurrence relation

$$
\mathrm{V}_{\mathrm{n}+1}=2 \mathrm{~V}_{\mathrm{n}}+\mathrm{V}_{\mathrm{n}-1} ; \mathrm{n} \geq 1 \text { with } \mathrm{V}_{0}=2, \mathrm{~V}_{1}=2
$$

In view of (1.1) we define a new class of numbers

$$
\mathrm{F}_{\mathrm{n}, \mathrm{k}}=(-1)^{\mathrm{n}-\mathrm{k}}\left(\begin{array}{c}
2 \mathrm{n}+1 \\
\mathrm{n}-\mathrm{k}
\end{array}\right) \mathrm{V}_{2 \mathrm{k}+1} \text { where } \mathrm{n} \text { is any non-negative integer and }
$$

$0 \leq \mathrm{k} \leq \mathrm{n}$.

Also these numbers generalize the Catalan numbers in a non trivial way.

The catalan numbers $\mathrm{C}_{\mathrm{n}}$ are defined by means of the generating relations ([3],p.82)

$$
\sum_{n=0}^{\infty} C_{n} t^{n}=\frac{1-\sqrt{1-4 t}}{2 t}
$$

Or by the explicit formula ([3],p.101)

$$
C_{n}=\frac{1}{n+1}\left(\begin{array}{c}
2 n \\
n
\end{array}\right)
$$

The following relationship is obvious

$$
\mathrm{C}_{\mathrm{n}}=\frac{(-1)^{\mathrm{n}}}{2} \quad \frac{\mathrm{F}_{\mathrm{n}, 0}}{2 \mathrm{n}+1}
$$

As usual $(\alpha)_{\mathrm{n}}$ is Pochhammer's symbol and is defined by

$$
(\alpha)_{n}=\left\{\begin{array}{ccc}
1 & \text { if } & n=0 \\
\alpha(\alpha+1) \ldots . .(\alpha+n-1), & \text { for all } & n \in\{1,2,3 \ldots \ldots . .
\end{array}\right\}
$$

${ }_{2} \mathrm{~F}_{1}$ will denote the hypergeometric function defined by 


$$
2 F_{1}\left[\begin{array}{c}
a, b ; \\
x \\
c ;
\end{array}\right]=\sum_{n=0}^{\infty} \frac{(a)_{n}\left(b_{n}\right)}{(c)_{n}} \frac{x^{n}}{n !} ; c \neq 0,1,-2,---
$$

The Jacobi Polynomials are defined by

$$
\mathrm{P}_{\mathrm{n}}^{(\alpha, \beta)}(\mathrm{x})=\frac{(1+\beta)_{\mathrm{n}}}{\mathrm{n} !}\left(\frac{\mathrm{x}-1}{2}\right)^{\mathrm{n}} 2 \mathrm{~F}_{1}\left[\begin{array}{ll}
-\mathrm{n},-\alpha-\mathrm{n} ; \\
1+\beta ; & \frac{\mathrm{x}+1}{\mathrm{x}-1}
\end{array}\right]
$$

\section{(1.9) In [1] A.K.Agarwal studied a new kind ofnumbers}

The New kind of Numbers are defined as $\mathrm{f}(\mathrm{n}, \mathrm{k})=(-1)^{\mathrm{n}-\mathrm{k}}\left(\begin{array}{c}2 \mathrm{n}+1 \\ \mathrm{n}-\mathrm{k}\end{array}\right) \mathrm{L}_{2 \mathrm{k}+1} ; 0 \leq \mathrm{k} \leq \mathrm{n}$

and $\mathrm{n}$ is a non-negative integers and $\mathrm{L}_{2 \mathrm{k}+1}$ is a Lucas number of order $2 \mathrm{k}+1$.

These new kind of numbers have the interesting property that

$$
\sum_{k=0}^{n} f(n, k)=1
$$

Theorem $1 \quad \sum_{\mathrm{k}=0}^{\mathrm{n}} \mathrm{F}_{\mathrm{n}, \mathrm{k}}=2^{2 \mathrm{n}+1}$

Proof : Let $\mathrm{n}$ be an odd positive integer and $\alpha, \beta$ be the roots of $\mathrm{x}^{2}=2 \mathrm{x}+1$ as defined in (1.1) Then from the binomial expansion of $(\alpha+\beta)^{\mathrm{n}}$ we get

$$
2^{\mathrm{n}}=\mathrm{V}_{1}-\left(\begin{array}{c}
\mathrm{n} \\
1
\end{array}\right) \mathrm{V}_{\mathrm{n}-2}+\left(\begin{array}{c}
\mathrm{n} \\
2
\end{array}\right) \mathrm{V}_{\mathrm{n}-4} \ldots+(-1)^{\frac{\mathrm{n}-1}{2}}\left(\begin{array}{c}
\mathrm{n} \\
\frac{\mathrm{n}-1}{2}
\end{array}\right) \mathrm{V}_{1}
$$

where $\mathrm{V}_{\mathrm{n}}$ is defined as in (1.1) Setting $\mathrm{n}=2 \mathrm{~m}+1$ in (1.11) we obtain

$$
2^{2 \mathrm{~m}+1}=\sum_{\mathrm{k}=0}^{\mathrm{m}}(-1)^{\mathrm{m}-\mathrm{k}}\left(\begin{array}{c}
2 \mathrm{~m}+1 \\
\mathrm{~m}-\mathrm{k}
\end{array}\right) \mathrm{V}_{2 \mathrm{k}+1}
$$

Remark 1 :Theorem 1 is analogues with the following property of Sterling numbers of the first kind [see [10],(6) p. 145]

$$
\sum_{\mathrm{k}=0}^{\mathrm{m}} \mathrm{S}_{\mathrm{n}}^{\mathrm{k}}=0
$$

Remark 2: Also theorem 1 is analogues with (1.10)

II.

. Table for $\mathrm{F}_{\mathrm{n}, \mathrm{k}}$

\begin{tabular}{|l|l|l|l|l|l|l|l|l|}
\hline $\mathrm{n} / \mathrm{k}$ & 0 & 1 & 2 & 3 & 4 & 5 & 6 & 7 \\
\hline 0 & 2 & & & & & & & \\
\hline 1 & -6 & 14 & & & & & & \\
\hline 2 & 20 & -70 & 82 & & & & & \\
\hline 3 & -70 & 294 & -574 & 478 & & & & \\
\hline 4 & 252 & -1176 & 2952 & -4302 & 2786 & & & \\
\hline 5 & -924 & 4620 & -13530 & 26290 & -30646 & 16238 & & \\
\hline
\end{tabular}

\section{Recurrence Relations}

(4.1) $\quad(\mathrm{n}+\mathrm{k}+2)(\mathrm{n}+\mathrm{k}+1) \mathrm{F}_{\mathrm{n}, \mathrm{k}+1}+6(\mathrm{n}-\mathrm{k})(\mathrm{n}+\mathrm{k}+1) \mathrm{F}_{\mathrm{n}, \mathrm{k}}+(\mathrm{n}-\mathrm{k}+1)$

(n-k) $\mathrm{F}_{\mathrm{n}, \mathrm{k}-1}=0 \quad$ for $\mathrm{k} \geq 1$ 
(4.2) $\quad \mathrm{F}_{\mathrm{n}+\mathrm{r}, \mathrm{k}}=(-1)^{\mathrm{r}} \frac{(2 \mathrm{n}+2)_{2 \mathrm{r}}}{(\mathrm{n}-\mathrm{k}+1)_{\mathrm{r}}(\mathrm{n}+\mathrm{k}+2)_{\mathrm{r}}} \mathrm{F}_{\mathrm{n}, \mathrm{k}}$

where $r$ is a non negative integer.

Proof of (4.1)

The sequence $\left\{\mathrm{V}_{2 \mathrm{k}+1}\right\}$ satisfies the following recurrence relations

(4.1.1) $\quad \mathrm{V}_{2 \mathrm{n}+3}=6 \mathrm{~V}_{2 \mathrm{k}+1}-\mathrm{V}_{2 \mathrm{k}-1}$

Now using the definition (1.2) and (4.1.1) we arrive at (4.1).

Proof of (4.2)

By definition, we have

$$
\mathrm{F}_{\mathrm{n}, \mathrm{k}}=(-1)^{\mathrm{n}-\mathrm{k}}\left(\begin{array}{c}
2 \mathrm{n}+1 \\
\mathrm{n}-\mathrm{k}
\end{array}\right) \mathrm{V}_{2 \mathrm{k}+1}
$$

And

$$
\mathrm{F}_{\mathrm{n}+1, \mathrm{k}}=(-1)^{\mathrm{n}-\mathrm{k}+1}\left(\begin{array}{c}
2 \mathrm{n}+3 \\
\mathrm{n}-\mathrm{k}+1
\end{array}\right) \mathrm{V}_{2 \mathrm{k}+1}
$$

Eliminating $\mathrm{V}_{2 \mathrm{k}+1}$ we have

$$
\mathrm{F}_{\mathrm{n}+1, \mathrm{k}}=(-1) \frac{(2 \mathrm{n}+3)(2 \mathrm{n}+2)}{(\mathrm{n}-\mathrm{k}+1)(\mathrm{n}+\mathrm{k}+2)} \mathrm{F}_{\mathrm{n}, \mathrm{k}}
$$

Proceeding in similar manner and using (1.6) we arrive at (4.2)

\section{Generating Relations}

$$
\sum_{\mathrm{n}=0}^{\infty} \frac{\mathrm{F}_{\mathrm{n}+\mathrm{k}, \mathrm{k}}}{2(\mathrm{k}+\mathrm{n})+1} \mathrm{y}^{\mathrm{n}}=\frac{\mathrm{x}^{2 \mathrm{k}+1}}{2 \mathrm{k}+1} \mathrm{~V}_{2 \mathrm{k}+1}
$$

$$
\sum_{n=0}^{\infty} F_{n+k, k} y^{n}=\frac{x^{2 k+2}}{2-x} V_{2 k+1}
$$

Where $\mathrm{y}=(1-\mathrm{x}) \mathrm{x}^{-2}$

Proof of (5. 1)

Here we shall use the identity

(5. 1.1) $\sum_{n=0}^{\infty} \frac{\alpha}{\alpha+n \beta}\left(\begin{array}{c}\alpha+n \beta \\ n\end{array}\right) y^{n}=x^{\alpha} ; y=(x-1) x^{-\beta}$

(see[3]. P. 147)

Setting $\alpha=2 \mathrm{k}+1$ and $\beta=2$ in (5.1.1) we arrive at (5.1)

Proof of (5. 2)

Here we shall use the identity

$$
\sum_{n=0}^{\infty}\left(\begin{array}{c}
\alpha+n \beta \\
n
\end{array}\right) y^{n}=\frac{x^{\alpha+1}}{(1-\beta) x+\beta} ; y=(x-1) x^{-\beta}
$$

(see[3]. P. 147)

Setting $\alpha=2 \mathrm{k}+1$ and $\beta=2$ we arrive at (5.2)

\section{Remark 3}


For $\mathrm{k}=0 ;(5.1)$ and (1.4) yields (1.3)

Remark 4 For $\mathrm{k}=0$; (5.2) yields the following generating relation for the Catalan numbers

$$
\sum_{n=0}^{\infty}(2 n+1) C_{n} y^{n}=\frac{x^{2}}{2-x} ; y=(x-1) x^{-2}
$$

Theorem ( 5.3) : Let $F_{n, k}$ be defined as in (1.2) then

$$
\begin{aligned}
& \sum_{n=0}^{\infty} \sum_{k=0}^{\infty} F_{n+k, k} y^{n+k}=\frac{2 x^{3}}{(2-x)\left(x^{2}-8 x+8\right)} \\
& \sum_{n=0}^{\infty} \sum_{k=0}^{\infty} \frac{F_{n+k, k}}{2(n+k)+1} y^{n+k}=x_{4}\left[1, \frac{1}{2} ; \frac{3}{2}, \frac{1}{2} ; 1-x, 2(1-x)\right]
\end{aligned}
$$

Where $\mathrm{F}_{4}$ is Appell's double hypergeometric function of fourth kind defined by (see[4],p.14)

$$
\mathrm{F}_{4}\left[\mathrm{a}, \mathrm{b} ; \mathrm{c}, \mathrm{c}^{\prime} ; \mathrm{x}, \mathrm{y}\right]=\sum_{\mathrm{m}=0}^{\infty} \sum_{\mathrm{n}=0}^{\infty} \frac{(\mathrm{a})_{\mathrm{m}+\mathrm{n}}(\mathrm{b})_{\mathrm{m}+\mathrm{n}}}{(\mathrm{c})_{\mathrm{m}}\left(\mathrm{c}^{1}\right)_{\mathrm{n}}} \frac{\mathrm{x}^{\mathrm{m}}}{\mathrm{m} !} \frac{\mathrm{y}^{\mathrm{n}}}{\mathrm{n} !}
$$$$
\sqrt{|\mathrm{x}|}+\sqrt{|\mathrm{y}|}<1
$$

Proof of (5.3.1):

The numbers $\left\{\mathrm{V}_{2 \mathrm{k}+1}\right\}$ Satisfy the following generating relation

$$
\sum_{\mathrm{k}=0}^{\infty} \mathrm{V}_{2 \mathrm{k}+1} \mathrm{x}^{\mathrm{k}}=\frac{2+2 \mathrm{x}}{\mathrm{x}^{2}-6 \mathrm{x}+1} ;|\mathrm{x}|<1
$$

Using the definition of $\mathrm{F}_{\mathrm{n}, \mathrm{k}}$ we have

$$
\sum_{n=0}^{\infty} \sum_{k=0}^{\infty} F_{n+k, k} y^{n+k}=\sum_{k=0}^{\infty} V_{2 k+1} y^{k} \sum_{n=0}^{\infty}\left(\begin{array}{c}
2 n+2 k+1 \\
n
\end{array}\right)(-y)^{n}
$$

Now summing the inner series with the help of (5.2.1) and using (5.3.4) we arrive at (5.3.1)

Proof of (5.3.2):

From the definition of the sequence $\left\{V_{n}\right\}$ we have.

$$
\mathrm{V}_{\mathrm{n}}=(1+\sqrt{2})^{\mathrm{n}}+(1-\sqrt{2})^{\mathrm{n}}
$$

Which allows us to use the identity

$$
{ }_{2} F_{1}\left[\begin{array}{c}
\frac{a}{2}, \frac{a}{2}+\frac{1}{2} ; \\
\frac{1}{2} ;
\end{array}\right]=\frac{1}{2}\left\{(1-z)^{-a}+(1+z)^{a}\right\}
$$

Setting $\mathrm{a}=-\mathrm{n}, \mathrm{z}=\sqrt{2}$ we see that

$$
{ }_{2} F_{1}\left[\begin{array}{c}
-\frac{\mathbf{n}}{2},-\frac{\mathbf{n}}{2}+\frac{1}{2} ; \\
2 \\
\frac{1}{2} ;
\end{array}\right]=\frac{1}{2}\left\{(1-\sqrt{2})^{\mathbf{n}}+(1+\sqrt{2})^{\mathbf{n}}\right\}
$$

Comparing (5.3.5) and (5.3.6) we get

$$
\mathbf{V}_{\mathbf{n}}=2_{2} \mathbf{F}_{1}\left[\begin{array}{rr}
-\frac{\mathbf{n}}{2}-\frac{1}{2},-\mathbf{n} & ; \\
\frac{1}{2} ; & 2
\end{array}\right]
$$


For odd $\mathrm{n}$ we have

(5.3.7)

$$
\mathrm{V}_{2 \mathrm{n}+1}=2{ }_{2} \mathrm{~F}_{1}\left[\begin{array}{cc}
-\mathbf{n}-\frac{1}{2},-\mathbf{n} ; \\
\frac{1}{2} ;
\end{array}\right]
$$

Again in (1.7) setting $\alpha=\frac{1}{2}, \beta=-\frac{1}{2}$ and $x=3$ we see that

$$
\mathbf{V}_{2 \mathrm{n}+1}=\frac{\mathbf{n} !}{\left(\frac{1}{2}\right)_{\mathrm{n}}} \rho_{\mathrm{n}}^{\left(\frac{1}{2},-\frac{1}{2}\right)}(3)
$$

Again in (1.8) setting $\gamma=1, \delta=\frac{1}{2}, \alpha=\frac{1}{2}, \beta=-\frac{1}{2}$ and $x=3$ we get

$$
\begin{aligned}
& \sum_{n=O}^{\infty} \frac{(1)_{n}\left(\frac{1}{2}\right)_{n}}{\left(\frac{3}{2}\right)_{n}\left(\frac{1}{2}\right)_{n}} \rho_{n}^{\left(\frac{1}{2},-\frac{1}{2}\right)}(3) t^{n}=F_{4}\left[1, \frac{1}{2} ; \frac{3}{2}, \frac{1}{2} ; t, 2 t\right] \\
& \Longrightarrow \sum_{n=0}^{\infty} \frac{n !\left(\frac{1}{2}\right)_{n}}{\left(\frac{1}{2}\right)_{n}(2 n+1)\left(\frac{1}{2}\right)_{n}} \rho_{n}^{\left(\frac{1}{2},-\frac{1}{2}\right)}(3) t^{n}=F_{4}\left[1, \frac{1}{2} ; \frac{3}{2}, \frac{1}{2} ; t, 2 t\right] \\
& \Rightarrow \sum_{\mathrm{n}=0}^{\infty} \frac{\mathrm{V}_{2 \mathrm{n}+1}}{2 \mathrm{n}+1} \mathrm{t}^{\mathrm{n}}=\mathrm{F}_{4}\left[1, \frac{1}{2} ; \frac{3}{2}, \frac{1}{2} ; \mathrm{t}, 2 \mathrm{t}\right]
\end{aligned}
$$

Now, Starting with L.H.S. we get

$$
\begin{gathered}
\sum_{n=0}^{\infty} \sum_{k=0}^{\infty} \frac{F_{n+k, k}}{2(n+k)+1} y^{n+k}=\sum_{k=0}^{\infty} V_{2 k+1} y^{k} \\
\sum_{n=0}^{\infty} \frac{1}{2(n+k)+1}\left(\begin{array}{c}
2 n+2 k+1 \\
n
\end{array}\right)(-y)^{n}
\end{gathered}
$$

Now summing the inner series with the help of (5.1.1) we get

$$
\begin{aligned}
& \sum_{\mathrm{n}=0}^{\infty} \sum_{\mathrm{k}=0}^{\infty} \frac{\mathrm{F}_{\mathrm{n}+\mathrm{k}}}{2(\mathrm{n}+\mathrm{k})+1} \mathrm{y}^{\mathrm{n}+\mathrm{k}}=\sum_{\mathrm{k}=0}^{\infty} \mathrm{V}_{2 \mathrm{k}+1} \mathrm{y}^{\mathrm{k}} \frac{\mathrm{x}^{2 \mathrm{k}+1}}{2 \mathrm{k}+1} \\
& =\mathrm{x} \sum_{\mathrm{k}=0}^{\infty} \frac{\mathrm{V}_{2 \mathrm{k}+1}}{2 \mathrm{k}+1}\left(\mathrm{x}^{2} \mathrm{y}\right)^{\mathrm{k}} \\
& =\mathrm{xF}_{4}\left[1, \frac{1}{2} ; \frac{3}{2} ; \frac{1}{2} ; 1-\mathrm{x}, 2(1-\mathrm{x})\right]
\end{aligned}
$$

\section{Summation Formulae}

$$
\sum_{m=0}^{n-1}\left\{F_{n+k-m-1, k}+\frac{V_{2 k+1}}{V_{2 k-1}} F_{n+k-m-1, k-1}\right\} F_{m, 0}=2 F_{n+k, k}
$$

$\mathrm{F}_{\mathrm{n}, 0} \mathrm{~V}_{2 \mathrm{k}+1} ; \mathrm{k} \geq 1$

$$
\sum_{m=0}^{n-1} \frac{F_{m, o}}{2 m+1} \frac{F_{n-m-1, o}}{2(n-m)-1}=\frac{2 F_{n, 0}}{2 n+1}
$$




$$
\begin{aligned}
& \sum_{m=0}^{n} \frac{n-2 m(k+1)}{(2 m+1)\{2(n+k-m)+1\}} F_{m, 0} F_{n+k-m, k}=0 \\
& \sum_{m=0}^{n}(-1)^{k}\left[\frac{F_{n, n-k}}{V_{2(n-k)+1}}+\frac{F_{n, n-k+1}}{V_{2(n-k)+3}}\right]=(2 n+1) C_{n}
\end{aligned}
$$

\section{Proof of (6.1):}

Replacing k by k-1 in (5.2) we obtain

$$
\text { (6.1.1) } \sum_{n=0}^{\infty} F_{n+k-1, k-1} y^{n}=\frac{x^{2 k}}{2-x} V_{2 k-1}
$$

Again putting $\mathrm{k}=0$ in (5.2) we obtain

(6.1.2) $\sum_{n=0}^{\infty} F_{n, 0} y^{n}=\frac{x^{2 k}}{2-x}$

From (5. 2) and (6.1.1) we obtain.

$$
\begin{aligned}
& \sum_{n=0}^{\infty} F_{n+k, k} y^{n}=\frac{V_{2 k+1}}{V_{2 k-1}} x^{2} \sum_{n=0}^{\infty} F_{n+k-1, k-1} y^{n} \\
& \Rightarrow \frac{1}{2-x} \sum_{n=0}^{\infty} F_{n+k, k} y^{n}=\frac{V_{2 k+1}}{V_{2 k-1}} \frac{x^{2}}{2-x} \sum_{n=0}^{\infty} F_{n+k-1, k-1} y^{n} \\
& \Rightarrow 2\left(1-\frac{x^{2} y}{2-x}\right) \sum_{n=0}^{\infty} F_{n+k, k} y^{n}=\frac{V_{2 k+1}}{V_{2 k-1}} \frac{2 x^{2}}{2-x} \sum_{n=0}^{\infty} F_{n+k-1, k-1} y^{n} \\
& \Rightarrow\left[2-\sum_{m=0}^{\infty} F_{m, 0} y^{m+1}\right] \sum_{n=0}^{\infty} F_{n+k, k} y^{n} \\
& \quad=\frac{V_{2 k+1}}{V_{2 k-1}} \sum_{m=0}^{\infty} F_{m, 0} y^{m} \sum_{n=0}^{\infty} F_{n+k-1, k-1} y^{n}
\end{aligned}
$$

Now equating the coefficients of $\mathrm{y}^{\mathrm{n}}$ we arrive at (6.1)

\section{Proof of (6.2)}

Putting $\mathrm{k}=0$ in (5.1) we obtain

$$
\begin{aligned}
& \sum_{\mathrm{n}=0}^{\infty} \frac{\mathrm{F}_{\mathrm{n}, 0}}{2 \mathrm{n}+1} \mathrm{y}^{\mathrm{n}}=2 \mathrm{x} \\
\Rightarrow & \sum_{\mathrm{n}=0}^{\infty} \frac{\mathrm{F}_{\mathrm{n}, 0}}{2 \mathrm{n}+1} \mathrm{y}^{\mathrm{n}}=2\left(1-\mathrm{x}^{2} \mathrm{y}\right) \\
= & 2-\frac{\mathrm{y}}{2} \sum_{\mathrm{m}=0}^{\infty} \frac{\mathrm{F}_{\mathrm{m}, 0}}{2 \mathrm{~m}+1} \mathrm{y}^{\mathrm{m}} \sum_{\mathrm{n}=0}^{\infty} \frac{\mathrm{F}_{\mathrm{n}, 0}}{2 \mathrm{n}+1} \mathrm{y}^{\mathrm{n}}
\end{aligned}
$$

Now equating the coefficients of $\mathrm{y}^{\mathrm{n}}$ we arrive at (6.2)

\section{Proof of (6.3):}

From (5.1) and (5. 2) we obtain.

$$
\begin{aligned}
& \sum_{n=0}^{\infty} F_{n+k, k} y^{n}=(2 k+1) \frac{x}{2-x} \sum_{n=0}^{\infty} \frac{F_{n+k, k}}{2(n+k)+1} y^{n} \\
& \Rightarrow 2 x \sum_{n=0}^{\infty} F_{n+k, k} y^{n}=(2 k+1) \frac{2 x^{2}}{2-x} \sum_{n=0}^{\infty} \frac{F_{n+k, k}}{2(n+k)+1} y^{n}
\end{aligned}
$$




$$
\begin{aligned}
& \Rightarrow \sum_{\mathrm{m}=0}^{\infty} \frac{\mathrm{F}_{\mathrm{m}, 0}}{2 \mathrm{~m}+1} \mathrm{y}^{\mathrm{m}} \sum_{\mathrm{n}=0}^{\infty} \mathrm{F}_{\mathrm{n}+\mathrm{k}, \mathrm{k}} \mathrm{y}^{\mathrm{n}} \\
& =(2 \mathrm{k}+1) \sum_{\mathrm{m}=0}^{\infty} \mathrm{F}_{\mathrm{m}, 0} \mathrm{y}^{\mathrm{m}} \sum_{\mathrm{n}=0}^{\infty} \frac{\mathrm{F}_{\mathrm{n}+\mathrm{k}, \mathrm{k}}}{2(\mathrm{n}+\mathrm{k})+1} \mathrm{y}^{\mathrm{n}}
\end{aligned}
$$

Now equating the coefficients of $\mathrm{y}^{\mathrm{n}}$ we arrive at (6.3)

Proof of (6.4):

Using the following identity (see[3] p.65)

$$
\left(\begin{array}{c}
2 \mathrm{n}+1 \\
\mathrm{n}
\end{array}\right)=\sum_{\mathrm{k}=0}^{\mathrm{n}}\left[\left(\begin{array}{c}
2 \mathrm{n}+1 \\
\mathrm{k}
\end{array}\right)-\left(\begin{array}{c}
2 \mathrm{n}+1 \\
\mathrm{k}-1
\end{array}\right)\right]
$$

We arrive at (6.4)

Remark 5 : In view of (1.5), (6.2) yields the following formula for Catalan numbers

$$
\sum_{\mathrm{m}=0}^{\mathrm{n}-1} \mathrm{C}_{\mathrm{m}} \mathrm{C}_{\mathrm{n}-\mathrm{m}-1}=\mathrm{C}_{\mathrm{n}}
$$

\section{References}

[1] A.K.Agarwal, "On a new kind of numbers", The Fibonacci Quarterly, Vol 28(3), ( 1990), 194-199

[2] C.Jordan. “Calculus of Finite Differences". New York : Chelsea, 1950.

[3] J.Riordan. “Cambinatorial Identities". Huntington. New York:Krieger, 1979

[4] E.D. Rainville. "Special Functions", New York : Macmillan, 1967. 